\title{
MULTIPLICACIÓN IN VITRO DE BROTES DE TRES VARIEDADES DE CALLAS (Zantedeschia sp.) EMPLEANDO SISTEMA DE INMERSIÓN TEMPORAL
}

\author{
Javier Sánchez, ${ }^{\circ}$ Marcos Daquinta e Iris Capote \\ Laboratorio de Células y Cultivo Tejidos, Centro de Bioplantas, Universidad de Ciego de Ávila, \\ C. P.: 69450,Cuba. ${ }^{\triangleright}$ mdaquinta@bioplantas.cu
}

\begin{abstract}
RESUMEN
$\mathrm{L}$ as Callas (Zantedeschia spp.) son plantas de la familia Araceae de gran interés como plantas ornamentales en maceteros y como flores de corte. Resulta de gran interés la propagación in vitro de las nuevas variedades obtenidas, por la diversidad de colores de sus flores. Cuando se desean introducir nuevos híbridos en el mercado, las técnicas de propagación tradicional son insuficientes, por lo que resultan de gran interés las técnicas de propagación in vitro. Con el objetivo de establecer un protocolo para la propagación in vitro más eficiente se evaluaron diferentes formas de cultivo (semi-sólido; líquido en movimiento e inmersión temporal), en la proliferación y calidad de los brotes. Se logró el establecimiento de un protocolo para la multiplicación in vitro de la Zantedeschia en Sistemas de Inmersión Temporal de mayor eficiencia biológica que los métodos convencionales de propagación (semi-sólido y liquido en movimiento).
\end{abstract}

Palabras claves: Zantedeschia, medio de cultivo, micropropagación, ornamentales.

\begin{abstract}
Callas (Zantedeschia spp.) belonging to Araceae family are ornamental plants with high commercial demand as plants in pot and cut flower. Traditional propagation techniques are not able to satisfy the fast introduction of new hybrids to the market. Thus, in vitro culture techniques are a useful tool for the propagation of new varieties with a wide range of colours. Aiming to establish a more efficient protocol for the in vitro propagation of callas, the effect of the way of culture (semisolid, shaken liquid media and Temporary Immersion) on shoots proliferation and quality, were evaluated. The protocol for in vitro propagation of Zantedeschia involving Temporary Immersion Systems showed higher biological efficiency than those through conventional methods (semi-solid and shaken liquid media).
\end{abstract}

Key words: Zantedeschia, culture medium, micropropagation, ornamentals.

\section{INTRODUCCIÓN}

$\mathrm{L}$ as callas son monocotiledóneas ornamentales originarias del Sur de África. Las especies de Zantedeschia (familia Araceae) y sus híbridos tienen flores con una variabilidad de colores que van desde rojo oscuro pasando por rosa, a naranja, amarillo y blanco (Funnell et al., 1998). Como flor cortada estas plantas son muy populares en todas partes del mundo, y están disponibles una multitud de cultivares e híbridos (Kritzinger et al., 1998)

La propagación del género Zantedeschia se realiza a través de semillas (en programas de mejoramiento), por separación de plantas (permite la formación del tubérculo y separar los tallos una vez que tienen raíz) y por división del tubérculo (practicada para incrementar el número y el tamaño de la floración). Sin embargo, los cultivos con división de tubérculos, están sujetos a la infección pudrición blanda de Erwinia, la cual causa serias pérdidas (Chen et al., 2000). Este género es muy susceptible a esta bacteria, que puede causar pérdidas de hasta el 100\% en la plantación (Etcheverría, 2002). La

Recibido: Octubre, 2009. Aceptado: Diciembre, 2009.

Publicado como ARTÍCULO en Ciencia y Tecnología 3(1): 1-5. 2010 literatura señala que el uso de la multiplicación in vitro permite una producción inmediata de plantas homogéneas libres de virosis (Funnell et al., 1998).

Se han propagado callas por micropropagación convencional en el medio de cultivo Murashige y Skoog (1962) con la utilización de reguladores de crecimiento, como benciladenina y tidiazuron (Chang et al., 2003) en Zantedeschia albomaculata. Las técnicas de cultivo de tejido se han desarrollado como una vía alternativa de plantación para la producción de tubérculos de Zantedeschia aethiopica (Cohen, 1981; Clemens y Welsh, 1993; Fang et al., 1999).

El efecto positivo de los Sistemas de Inmersión Temporal en la micropropagación de plantas se ha empleado con éxitos en la proliferación de meristemos, cultivo de microestacas, desarrollo de embriones a partir de callos, así como también en la germinación y conversión de embriones somáticos. Por lo general, la calidad del desarrollo de los brotes es mejor que la obtenida con el empleo de medio líquido o semi-sólido (Santos et al., 2006).

Existen pocos trabajos de micropropagación de Zantedeschia en Sistemas de Inmersión Temporal, uno 
de los trabajos realizado para esta especie por Ruffoni et al,. (2002), logró tasas de multiplicación muy bajas, no variando cuando se evaluó el sistema convencional y los Sistemas de Inmersión Temporal (Ruffoni y Savona, 2005).

Se evaluaron diferentes formas de cultivo (semi-sólido; líquido en movimiento e inmersión temporal) con el objetivo de lograr un mayor coeficiente de propagación clonal en tres variedades de callas.

\section{Materiales y Métodos}

Ne utilizaron yemas activadas obtenidas de tubérculos previamente aviverados, se procedió a seleccionar las yemas brotadas que tuvieran $1-5 \mathrm{~cm}$ de tallo. Se realizó una desinfección con bicloruro de mercurio a $0.25 \%$ durante $10 \mathrm{~min}$, después del lavado con detergente comercial, y se estableció en el medio de cultivo de implantación compuesto por las sales de Murashige y Skoog (1962), $1 \mathrm{mg} \mathrm{L}^{-1}$ de tiamina, $100 \mathrm{mg} \mathrm{L}^{-1}$ de mio-inositol, $30 \mathrm{~g} \mathrm{~L}^{-1}$ de sacarosa y $2 \mathrm{mg} \mathrm{L}^{-1}$ de BA en la oscuridad. A las cuatro semanas se realizó el primer subcultivo. La temperatura de la cámara de penumbra fue de $25 \pm 2^{\circ} \mathrm{C}$. A los brotes con una altura de $2-3 \mathrm{~cm}$ se les realizó un corte longitudinal para romper la dominancia apical, y así los mismos quedaron divididos en dos partes, en algunos casos en dependencia del tamaño de los brotes y grosor de su base (mayor de $0.5 \mathrm{~cm}$ de tallo y base) se cortaron en cuatro secciones. Las partes seccionadas se colocaron en frascos de vidrio de 250 $\mathrm{mL}$ de capacidad con $25 \mathrm{~mL}$ de medio de cultivo MS + 1-4 mg L-1 BA (dependiendo de la variedad), y posteriormente fueron transferidos a la luz con $30 \mu \mathrm{mol} \mathrm{m} \mathrm{m}^{-2}$ $\mathrm{s}^{-1}$ de Flujo de Fotones Fotosintéticos.

Evaluación del efecto de la forma de cultivo en el coeficiente de multiplicación y calidad de los brotes de tres variedades comerciales de callas

Se subcultivaron brotes in vitro de callas (variedad Majestic Red, Golden Affair y Treasure) en un medio de cultivo MS con la adición de $4 \mathrm{mg} \mathrm{L}^{-1} \mathrm{BA}$ (Treassure), $2 \mathrm{mg} \mathrm{L}^{-1} \mathrm{BA}$ (Golden Affiar) y $1 \mathrm{mg} \mathrm{L}^{-1} \mathrm{BA}$ (Magistec Red), en diferentes formas de cultivo, semisólido, líquido en movimiento a 100 rpm y en Sistemas de Inmersión Temporal (SIT). Se utilizaron cinco explantes por frasco y tres repeticiones por tratamiento. La frecuencia de inmersión fue cada cuatro horas, por un tiempo de tres min (según resultados precedentes).

A las cuatro semanas se separaron grupos de tres brotes que fueron transferidos al medio de cultivo referido en el establecimiento, pero sin reguladores del crecimiento, durante otras cuatro semanas. En la multiplicación y el enraizamiento la temperatura de las cámaras de cultivo se mantuvo a $25 \pm 2^{\circ} \mathrm{C}$ y con $30 \mu \mathrm{mol} \mathrm{m}{ }^{-2}$ $\mathrm{s}^{-1}$ de Flujo de Fotones Fotosintéticos.

Los brotes con dos hojas expandidas y dos o más raíces se colocaron en bandejas con sustrato de cachaza $(100 \%)$ bajo condiciones de nebulización (cinco seg cada $30 \mathrm{~min}$ ) las primeras dos semanas, y al cabo de este tiempo se les retiró el cobertor de saran de $70 \%$ de sombra.

\section{Resultados y Discusión}

Evaluación del efecto de la forma de cultivo en el coeficiente de multiplicación y calidad de los brotes de tres variedades comerciales de callas

$E^{n}$ el Cuadro 1 se observa el comportamiento de la variedad Treasure en cuanto al número de hojas por brote, altura y coeficiente de multiplicación en dependencia de la forma de cultivo. En el Sistema de Inmersión Temporal se obtuvo coeficientes de multiplicación de 7.93, con diferencias significativas con respecto al medio de cultivo semisólido y líquido en movimiento. Sin embargo, en cuanto a las variables de calidad evaluadas, no se encontraron diferencias significativas entre las tres formas de cultivo.

Cuadro 1. Efecto de la forma de cultivo sobre el crecimiento y multiplicación de calla variedad Treasure a los 45 días de cultivo

\begin{tabular}{lcccc}
\hline \multicolumn{1}{c}{ Forma de cultivo } & $\begin{array}{c}\text { Número de } \\
\text { hojas/brote }\end{array}$ & Altura & $\begin{array}{c}\text { Coeficiente } \\
\text { Multiplicación }\end{array}$ \\
\hline Semi-sólido & 1.91 & 2.53 & 3.63 & $\mathrm{~b}$ \\
Líquido en movimiento & 1.86 & 3.33 & 3.96 & $\mathrm{~b}$ \\
SIT & 1.90 & 2.29 & 7.93 & $\mathrm{a}$ \\
\hline
\end{tabular}

*Medias con letras iguales no difieren (KW, C Dunnett, $\mathrm{p}<0.05$ ) 
Coeficiente de multiplicación: Número de explantes finales/Número de explantes iniciales

El comportamiento de la Zantedeschia variedad Golden Affair en cuanto al número de hojas por brote, altura y coeficiente de multiplicación en dependencia de la forma de cultivo, se presenta en el Cuadro 2. En esta variedad en el Sistema de Inmersión Temporal se obtuvo coeficientes de multiplicación de 10.66, con diferencias significativas con respecto al medio de cultivo semisólido y líquido en movimiento, incluso mayor al obtenido para la variedad Treasure. Al igual que la variedad Treasure, no se encontraron diferencias significativas en cuanto al número de hojas y altura de los brotes, aunque se encontró en esta variedad más hojas y mayor altura, características propias de cada variedad.

Cuadro 2. Efecto de la forma de cultivo sobre el crecimiento y multiplicación de calla variedad Golden Affair a los $\mathbf{4 5}$ días de cultivo

\begin{tabular}{lccc}
\hline \multicolumn{1}{c}{ Forma de cultivo } & $\begin{array}{c}\text { Número de } \\
\text { hojas/brote }\end{array}$ & Altura & \multicolumn{2}{c}{$\begin{array}{c}\text { Coeficiente } \\
\text { Multiplicación }\end{array}$} \\
\hline Semi-sólido & 2.39 & 4.43 & $6.50 \mathrm{~b}$ \\
Líquido en movimiento & 2.28 & 5.67 & $3.70 \mathrm{c}$ \\
SIT & 2.13 & 4.71 & $10.66 \quad \mathrm{a}$ \\
\hline
\end{tabular}

*Medias con letras iguales no difieren (KW, ANOVA, C Dunnett, $\mathrm{p}<0.05$ )

En el Cuadro 3 se presenta la respuesta de la variedad Magister Red en cuanto al número de hojas por brote, altura y coeficiente de multiplicación en dependencia de la forma de cultivo. En el Sistema de Inmersión Temporal se obtuvo coeficientes de multiplicación de 11.3, con diferencias significativas con respecto al medio de cultivo semisólido y líquido en movimiento. Por otra parte, al igual que en las variedades Treasure y Golden Affair no se encontraron diferencias significativas en cuanto al número de hojas y altura de los brotes.

Cuadro 3. Efecto de la forma de cultivo sobre el crecimiento y multiplicación de calla variedad Magister Red a los $\mathbf{4 5}$ días de cultivo

\begin{tabular}{lcccc}
\hline \multicolumn{1}{c}{ Forma de cultivo } & $\begin{array}{c}\text { Número de } \\
\text { hojas/brote }\end{array}$ & Altura & \multicolumn{2}{c}{$\begin{array}{c}\text { Coeficiente } \\
\text { Multiplicación }\end{array}$} \\
\hline Semi-sólido & 2.20 & 4.15 & 7.23 & $\mathrm{~b}$ \\
Líquido en movimiento & 2.39 & 5.28 & 4.26 & $\mathrm{c}$ \\
SIT & 2.02 & 4.51 & 11.30 & $\mathrm{a}$ \\
\hline
\end{tabular}

*Medias con letras iguales no difieren (KW, C Dunnett, $\mathrm{p}<0.05$ )

Los coeficientes obtenidos en medio de cultivo semi-sólido son más altos que los obtenidos por Chang et al. (2003) en trabajos realizados con Zantedeschia albomaculata, quienes alcanzaron un coeficiente de multiplicación de 3.8, en el medio de cultivo semi-sólido. Por su parte Ruffoni y Savona (2005) lograron en Sistemas de Inmersión Temporal tipo RITA sólo 3.44 de coeficiente de multiplicación en la especie Zantedeschia aethiopica, incluso con una concentración de citoquinina similar a la empleada en la variedad Treasure en este trabajo ( $\left.4 \mathrm{mg} \mathrm{L}^{-1} \mathrm{BA}\right)$. La calidad visual de los brotes obtenidos en SIT fue mejor al medio de cultivo semi-sólido y al líquido, los cuales mostraron síntomas de clorosis (datos no mostrados). La forma de cultivo en inmersión temporal incrementó significativamente el número de brotes en todas las variedades evaluadas.

Las respuestas morfogenéticas de los explantes ante las propiedades físicas del medio de cultivo se relaciona con el potencial hídrico (osmótico y matricial) y es indicativo de la disponibilidad de agua que tienen las plantas en el cultivo in vitro (Debergh et al., 1981; George, 1993).

La efectividad de la técnica de inmersión temporal en el aumento de la proliferación y calidad de los brotes ha sido señalada en otras especies de plantas (Etienne y Berthouly, 2002). También el empleo de esta técnica ha sido efectiva en la proliferación de otras Aráceas ornamentales (Daquinta et al., 2007) 
Existen varios ejemplos en la literatura que fundamentan el efecto favorable de la técnica de inmersión temporal en la calidad de los brotes. Brotes de fresa (Fragaria x ananassa) propagados en inmersión temporal incrementaron la masa fresca y fueron de mayor altura que los cultivados en medio de cultivo semi-sólido (Krueger et al., 1991, Debnath, 2008). La inmersión temporal incrementó la calidad morfológica de los brotes en bananos (De Feria et al., 2005), caña de azúcar (De Feria et al., 2002), Caladium (Daquinta et al., 2007) y Phalaenopsis (Pisowotzki et al., 2008) en comparación con el cultivo en medio semi-sólido.

Entre las ventajas que ofrece esta técnica de cultivo, está el estrecho contacto del medio de cultivo con los explantes durante cada inmersión, estimula y facilita una forma más eficiente de suministro de los nutrientes y reguladores del crecimiento, en comparación con el cultivo en medio convencional (Etienne y Berthouly, 2002).

Mehrotra et al. (2007) han destacado la eficiencia de los sistemas de cultivo liquido sobre la micropropagación convencional, permitiendo el mejor crecimiento de brotes y raíces. También los mismos autores señalan que la óptima producción de plantas en estos sistemas depende del mejor entendimiento de las respuestas fisiológicas y bioquímicas al cultivo en el microambiente y a la optimización de las condiciones de cultivo físicas y químicas para el control de la morfogénesis de las plantas en los sistemas de cultivo líquido.

Por otra parte, cuando los tiempos de inmersión son cortos, la mayoría del tiempo los explantes están recubiertos de una película de medio de cultivo líquido y de esta forma se evita la desecación de los mismos. La resistencia a la difusión de gases es baja y existe una mínima ruptura del intercambio gaseoso entre los tejidos y la atmósfera, por esta razón dentro del vaso de cultivo se renueva el ambiente gaseoso en intervalos regulares de tiempo. Además, la agitación por el flujo de aire durante la fase de inmersión causa expansión de los tejidos y se facilita un mayor contacto de éstos con el medio de cultivo (Teisson y Alvard, 1995).

De Feria et al. (2002), demostraron en diferentes variedades de caña de azúcar (Saccharum spp.), que el cultivo en inmersión temporal estimuló la formación de brotes y el crecimiento de los mismos. La tasa de multiplicación obtenida en esta forma de cultivo fue seis veces mayor que la obtenida en los protocolos convencionales. Resultados similares han sido obtenidos por Escalona et al. (1999) en el cultivo de la piña (Ananas comosus) utilizando similar Sistema de Inmersión Temporal. En este cultivo, la inmersión temporal estimuló la formación de brotes, conjuntamente con la masa fresca y seca de los mismos. Roels et al. (2005) lograron con el empleo de esta forma de cultivo aumentar la tasa de multiplicación, así como la calidad morfológica de los brotes en el plátano CEMSA $3 / 4$. Aunque en plantas ornamentales los resultados han sido mas reservados, Daquinta et al. (2007) lograron un coeficiente de multiplicación en este sistema 12 veces superior que el obtenido en los medios de cultivo convencionales para otra especie de la familia Aracea (Caladium x hortulanum).

Autores como Hempling y Preil (2005) al trabajar con Phalaenopsis en SIT plantearon que al realizarse un mayor número de inmersiones al día se favoreció la ventilación y el intercambio de gases como el etileno y el $\mathrm{CO}_{2}$ que al acumularse limitan la multiplicación de los brotes, razón por la cual es indispensable determinar la frecuencia de inmersión adecuada para cada especie.

\section{Conclusiones}

Tos Sistemas de Inmersión Temporal resultaron suLeriores a las demás formas de cultivo evaluadas, en cuanto al coeficiente de multiplicación obtenido. Se obtuvo un incremento de 4.3, 4.16 y 4.07 de las tasas de proliferación en los Sistemas de Inmersión Temporal en las variedades Treasure, Golden Affair y Majestic Red. Con la utilización de los Sistemas Inmersión Temporal en las tres variedades de callas se logró obtener un aumento del coeficiente de multiplicación con respecto al medio de cultivo semi-sólido y líquido en movimiento.

\section{Literatura Citada}

Chang, H., D. Chakrabarty, E. Hahn \& K. Paek. 2003. Micropropagaction of Calla Lily (Zantedeschia albomaculata) via in vitro shoot tip proliferation. In Vitro Cellular and Development Biology - Plant. 36:129-134.

Chen, J., M. Liu \& Y. Ho. 2000. Size on in vitro plantlets affects subsequent tuber production of acclimatized calla lily. HortScience. 35(2):290-292.

Clemens, J. \& T. Welsh. 1993. An overview of the New Zealand calla industry, research direction and year round tuber production. Acta Horticulturae 337:161-316.

Cohen, D. 1981. Micropropagation of Zantedeschia hybrids. Combined Proc. Int. Plant Pro. Soc. 31:312316.

Daquinta, M., O. Mosqueda, M. T. Gonzalez, R. Benega \& J. Á. Texeira da Silva. 2007. Shoot Proliferation of Caladium x hortulanum in a Temporary Immersion System. Floriculture and Ornamental Biotechnology. 1(1): 70-72. 
Debergh, P., Y. Harbaooui \& C. Lemeur. 1981. Mass propagation of globe artichoke (Cynara scolymus) evaluation of different hypotheses to overcome vitrification wtih special reference to water potential. Physiologia Plant. 53: 181-187.

Debnath, S. 2008. Developing a scale-up system for the in vitro multiplication of thidiazuron-induced strawberry shoots using a biorreactor. Canadian Journal of Plant Science. 88: 737-746.

De Feria, M., E. Jiménez y M. Chávez. 2002. Empleo de sistemas de inmersión temporal para la multiplicación in vitro de brotes de Saccharum spp. var. IBP 89-112. Biotecnología Vegetal. 2(3): 143-147.

De Feria, M., E. Quiala, M. Chávez, L. Molejón, E. Peralta, M. Hernández, A. Rodríguez, D. Mirabal y Y. Sánchez. 2005. Efecto de la frecuencia de inmersión y la disponibilidad de medio de cultivo en la multiplicación del cultivar híbrido FHIA-21 (Musa AAAB) en sistemas de inmersión temporal. Biotecnología Vegetal. 5(1): 9-15.

Escalona, M., J. C. Lorenzo, B. González, M. Daquinta, J. L. González, Y. Dejardins y C. G. Borroto. 1999. Pineapple (Ananas comosus (L). Merr) micropropagation in temporary immersion systems. Plant Cell Reports, 18(9): 723-748.

Etcheverría, P. 2002. Efecto de la densidad de sombra y del mulch en la producción y calidad de flores y túberos de Zantedeschia hybrida cv. Mango. Tesis Ingeniero Agrónomo, Universidad de la Frontera. $67 \mathrm{p}$.

Etienne, H. \& M. Berthouly. 2002. Temporary immersion systems in plant micropropagation. Plant Cell, Tissue and Organ Culture. 69: 215-231.

Fang, W. L., L. Xiong, Y. H. Qu \& S. P. Qu. 1999. Tissue culture of colored common calla lily. J. South West Agricultural University. 21: 423-426.

Funnell, K., E. Hewett, I. Warrington \& J. Pummer. 1998. Leaf mass portioning as a determinant of dry matter accumulation in Zantedeschia. Journal American Society Horticultural Science. 123(6): 973-979.

George, E. F. 1993. Plant propagation by tissue culture. Part 1. 2da Edition. Exergetics 1.

Hempfling, T., W. Preil. 2005. Application of a temporary immersion system in mass propagation of Phalaenopsis. In: Hvoslef-Eide AK, Preil W (Eds) Liquid Culture Systems for in Vitro Plant Propagation, Springer, Dordrecht, The Netherlands, pp 231-242
Kritzinger, E., R. Cansen Van Vuuren, B. Wood-War, I. Rong, M. Spreeth \& M. Slabbert. 1998. Elimination of external and internal contaminants in rhizomes of Zantedeschia aethiopica with commercial fungicides and antibiotics. Plant Cell, Tissue and Organ Culture 52: 61-65.

Krueger, S., C. Robacer, W. Simonton. 1991. Culture of Amelanchier grandiflora in a programmable micropropagation apparatus. Plan Cell, and Tissue and Organ Culture. 27: 219-226.

Mehotra, S., M. Goel, A. Kukreja \& B. Mishra. 2007. Efficiency of liquid culture systems over conventional micropropagation: A progress towards commercialization. African Journal of Biotechnology. 6(13): 1484-1492.

Murashige, T. \& F. Skoog. 1962. A revised medium for rapid growth and bioassays with tobacco tissue culture. Physiologia Plantarum.15: 473-487.

Pisowotzki, C., K. Saare-Surminski \& R. Lieberei. 2008. Micropropagation of Phalaenopsis-hybrids in Temporary Immersion System (TIS). Effect of exudated phenolic substances on plant development. Propagation of Ornamental Plants. 8(1): 221-223

Roels, S., M. Escalona, I. Cejas, C. Noceda, R. Rodriguez, M. J. Cana, J. Sandoval \& P. Debergh. 2005. Optimizacion of plantain (Musa AAB) micropropagation by temporary immersion system. Plant Cell, Tissue and Organ Culture. 82: 57-66.

Ruffoni, B., M. Savona, S. Doveri, M. Pamato, M. Laura, M. Rabaglio \& M. Brea. 2002. Propagazione in vitro di un genotipo a taglia ridotta di Zantedeschia aethiopica. Proc VI Giornate Scientifiche SOI, Workshop: 75-76.

Ruffoni, B. \& M. Savona. 2005. The Temporary Immersion System (T.I.S.) for the Improvement of Micropropagatión of Ornamental Plants. Acta Horticulturae. 683: 445-449.

Santos, R., J. González-Olmedo, M. De Feria, M. Cornide y W. Torres. 2006. Biotecnología Vegetal. En colectivo de autores (eds.). Las Investigaciones Agropecuarias en Cuba, cien años después. La Habana, Cuba. Ed. Científico-Técnica, pp 207-221.

Teisson, C. \& D. Alvard. 1995. A new concept of plant in vitro cultivation liquid médium: Temporary Immersion. In: Terzi M, Cella R, Falavigna A (Eds). Current Issues in Plant Molecular and Cellular Biology. Pp 105-109 Kluwer Academic Publishers. 